\title{
Package-Based Description Logics - Preliminary Results
}

\author{
Jie Bao $^{1}$, Doina Caragea ${ }^{2}$, and Vasant G. Honavar ${ }^{1}$ \\ ${ }^{1}$ Artificial Intelligence Research Laboratory, Department of Computer Science, \\ Iowa State University, Ames, IA 50011-1040, USA \\ \{baojie, honavar\}@cs.iastate.edu \\ ${ }^{2}$ Department of Computing and Information Sciences \\ Kansas State University, Manhattan, KS 66506, USA \\ dcaragea@ksu.edu
}

Many representative applications on the semantic web, including collaborative ontology building, partial ontology reuse, selective knowledge hiding and distributed data management, call for modular ontologies,. However, although OWL allows using owl:imports to connect multiple ontologies, its current semantics requires all involved ontologies to have a single global semantics, thus providing only a syntactical solution to modularity. As a result, there is a growing interest in modular ontology languages such as Distributed Description Logics (DDL) 7 ] and $\mathcal{E}$-connections [8]. However, these proposals are also limited in expressivity and reasoning soundness 233 .

Package-based Description Logics (P-DL) [4] is aimed at solving several problems presented in existing approaches, by offering a tradeoff between the strong module disjointness assumption of DDL and $\mathcal{E}$-connections, and the complete overlapping of models required by the OWL importing mechanics. P-DL language features are aimed at providing fine-grained modular organization and controllable selective knowledge sharing of an ontology.

P-DL syntax adopts a selective "importing" approach that allows a subset of terms defined in one ontology module to be directly used in another module. In a P-DL ontology, an ontology is composed of a set of packages. A package can use terms defined other packages i.e., an existing package or some of the terms defined in an existing package can be imported into another package. Foreign terms can be used to construct local concepts.

P-DL also allows selective knowledge hiding in ontology modules to address the needs of privacy, copyright, security concerns in ontologies. P-DL supports scope limitation modifiers (SLM) that can be associated with terms and axioms defined in a package 4. A SLM (such as public and private) controls the visibility of the corresponding term or axiom to entities (e.g. a user, a reasoner) on the web, in particular, to other packages. Different from the encryption of ontology which is aimed at safe access of ontologies on a syntactic level, SLM in P-DL aims at knowledge hiding on a semantic level, where the hiding is partial, i.e., hidden parts of an ontology can be used in safe indirect inferences [5].

The semantic importing approach adopted by P-DL is different from the "linking" approach adopted by DDL and $\mathcal{E}$-Connections in that it partially relaxes 
the local model disjointness assumption of the other two formalisms. Such a relaxation enables P-DL to obtain stronger expressivity power. Concept bridge rules in DDL and $\mathcal{E}$-Connection links can be easily reduced to P-DL axioms 3 . P-DL also offers the possibility of avoiding semantic difficulties of DDL and $\mathcal{E}$ Connections. For example, knowledge in one P-DL package can be transitively reused by other packages. The answer to a P-DL reasoning problem is the same as that obtained by reasoning over the integrated ontology 3 .

The reasoning procedure for P-DL can be extended from existing DL tableau algorithms [1]. We adopt a tableau-based federated reasoning approach to strictly avoid reasoning with an integrated ontology, thus ensure the autonomy of constituting modules. The whole reasoning process is preformed by a federation of local reasoners, each for a specific package, to construct a collection of local tableaux instead of a single global tableau. The connection between local tableaux is enabled by a set of messages and a local tableau may share nodes with other local tableaux. It is shown that this approach can solve many reasoning difficulties presented in existing approaches [1].

P-DL provides language features needed for efficient collaborative construction of large, modular ontologies. We have developed COB-Editor [6] that provides 'proof of concept' of this approach. COB-Editor is a modular ontology editor that enables building biological ontologies such as Gene Ontology. The editor allows ontology developers to create a community-shared ontology server, with the support for concurrent browsing and editing of the ontology. Multiple users can work on the same ontology on different packages (through locking mechanisms), without inadvertent overwriting the work of others.

Work in progress includes reasoning for more expressive P-DL languages and with knowledge hiding, and improved collaborative ontology building tools.

Acknowledgement. This research is supported by grants from US NSF (0219699, 0639230) and NIH (GM066387).

\section{References}

1. J. Bao, D. Caragea, and V. Honavar. A distributed tableau algorithm for packagebased description logics. In the 2nd International Workshop On Context Representation And Reasoning (CRR 2006), co-located with ECAI 2006. 2006.

2. J. Bao, D. Caragea, and V. Honavar. Modular ontologies - a formal investigation of semantics and expressivity. In R. Mizoguchi, Z. Shi, and F. Giunchiglia (Eds.): Asian Semantic Web Conference 2006, LNCS 4185, pages 616-631, 2006.

3. J. Bao, D. Caragea, and V. Honavar. On the semantics of linking and importing in modular ontologies. In accepted by ISWC 2006 (In Press). 2006.

4. J. Bao, D. Caragea, and V. Honavar. Towards collaborative environments for ontology construction and sharing. In International Symposium on Collaborative Technologies and Systems (CTS 2006), pages 99-108. IEEE Press, 2006.

5. J. Bao and V. Honavar. Representing and reasoning with modular ontologies. In AAAI Fall Symposium on Semantic Web for Collaborative Knowledge Acquisition (SWeCKa 2006), Arlington, VA, USA, October 2006, 2006. 
6. J. Bao, Z. Hu, D. Caragea, J. Reecy, and V. Honavar. Developing frameworks and tools for collaborative building of large biological ontologies. In the 4 th International Workshop on Biological Data Management (BIDM'06), @ DEXA'06. 2006.

7. A. Borgida and L. Serafini. Distributed description logics: Directed domain correspondences in federated information sources. In CoopIS, pages 36-53, 2002.

8. B. C. Grau. Combination and Integration of Ontologies on the Semantic Web. PhD thesis, Dpto. de Informatica, Universitat de Valencia, Spain, 2005. 